\title{
ENT Manifestations of Gastroesophageal Reflux
}

\author{
Desiderio Passàli, $M D$, PhD, Giuseppe Caruso, $M D$, \\ and Francesco Maria Passàli, MD, PhD
}

\author{
Corresponding author \\ Desiderio Passàli, MD, PhD \\ Ear, Nose, and Throat Clinic, Policlinico "Le Scotte," \\ University of Siena, 53100, Siena, Italy. \\ E-mail: passali@unisi.it
}

Current Allergy and Asthma Reports 2008, 8:240-244

Current Medicine Group LLC ISSN 1529-7322

Copyright (C) 2008 by Current Medicine Group LLC

Gastroesophageal reflux (GER) has been associated with a variety of supraesophageal symptoms or diseases, including chronic cough, laryngeal disorders, rhinosinusitis, otitis media, and oral cavity lesions. In this article, we review the relationship between GER and ear, nose, and throat (ENT) symptoms. Data in the published literature are frequently conflicting. Only a few studies are controlled, and an evidence-based approach provides weak support for a causal association between GER and ENT manifestations. The GER diagnostic method should be standardized utilizing new parameters, and the definition and diagnostic accuracy of ENT pathologies also must be better specified. A firm connection remains controversial, and further randomized trials are needed.

\section{Introduction}

The role of gastroesophageal reflux (GER) in causing extraesophageal symptoms is well known. All the otorhinolaryngologic regions may be involved (ie, larynx, pharynx, nose, paranasal sinuses, and middle ear), including the oral and dental region. A possible pathogenetic mechanism is the direct action of GER on the respiratory mucosa and on the hypopharyngolaryngeal site. Some researchers have hypothesized a vagus-mediated reflex. Esophageal mucosa with intrinsic antireflux defenses can temporarily bear insults without anatomic modifications of the epithelium, whereas the respiratory mucosa is not resistant to acid injury even with limited length of exposure. Because no standard definition or diagnostic method exists for GER, all types of reflux (acid, nonacid, liquid, mixed, and air) detected by impedance changes probably should be considered in the diagnosis. The 24-hour double-probe $\mathrm{pH}$ monitoring (at esophageal and pharyngeal levels)-which is the most specific method for GER diagnosis-has been established as the gold standard to qualitatively and quantitatively evaluate esophageal acid exposure and to correlate symptoms to reflux. Recently, a wireless monitoring system was introduced as an alternative to double-probe $\mathrm{pH}$ monitoring $[1 \bullet \bullet]$.

The DeMeester score and its six parameters (supine reflux, upright reflux, total reflux, number of episodes, number of episodes > 5 minutes, and longest episode) are based exclusively on time of reflux: score and total time of reflux represent the most useful parameters. New parameters have been introduced for the evaluation of pathological GER, such as the area under the curve at $\mathrm{pH} 4$ (AUC $\mathrm{pH} 4$ ), the area under the $\mathrm{H}^{+}$curve $\left(\mathrm{AUCH}^{+}\right.$ $\mathrm{mmol} / \mathrm{L} / \mathrm{min}$ ), and the evaluation of short reflux or index of esophageal clearance (given as a percentage). According to our studies, $\mathrm{AUCH}^{+}$represents the best parameter to evaluate GER in adults and children, in both erosive and nonerosive GER, because it assures high specificity and sensibility $[2 \bullet \bullet]$. In this paper, we consider the ear, nose, and throat (ENT) manifestations related to GER.

\section{Laryngopharyngeal Manifestations}

GER into the larynx can cause posterior laryngeal inflammation, contact ulcers, and granulomas, which clinically improve with antireflux therapy. The term laryngopharyngeal reflux (LPR) indicates reflux that reaches the upper airways. At present, GER and LPR symptoms are believed to run in parallel, probably as two sides of the same coin, with LPR representing a supraesophageal manifestation of gastroesophageal reflux disease (GERD) [3,4•].

Reflux laryngitis is the most common clinical manifestation correlated to LPR. The presenting laryngeal symptoms include hoarseness, sore throat, throat clearing, and chronic cough. Endoscopic laryngeal examination is poorly specific with signs that may be minimal or absent: slight vocal cord erythema and edema, erythema of both arytenoids and posterior commissure, or increased mucosal secretion. In some advanced cases, the interarytenoid mucosa may be hypertrophic and laryngeal granulations may be present at the posterior third 
of the vocal folds. Laryngeal edema, more than posterior commissure hyperemia, seems to be the objective finding in conjunction with LPR. In this regard, the reflux finding score (RFS) and the reflux symptom index (RSI) are useful clinical tools. The RFS and the RSI have a highly significant correlation, and empiric pharmacologic therapy can be warranted with a diagnosis of LPR based

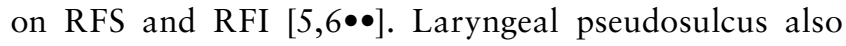
has been associated with LPR. According to Hickson et al. [7], pseudosulcus vocalis, which represents a pattern of edema on the ventral surface of the vocal cord, is an accurate prognostic indicator of LPR with a positive predictive value of $90 \%$. Belafsky et al. [8] reported a high correlation between pseudosulcus and $\mathrm{pH}$-documented LPR, with a sensitivity of $70 \%$ and specificity of $77 \%$. Based on these data, the pseudosulcus is suggestive but not pathognomonic for LPR. Recently, Joniau et al. [9] systematically reviewed the literature to identify all relevant articles on pharyngeal reflux (PR) events in normal controls and patients with reflux laryngitis. The authors noted the relatively low number of PR events in patients with clinically diagnosed reflux laryngitis and concluded that "in these times of evidence-based medicine, it seems difficult to defend reflux laryngitis as a mere clinical diagnosis, not taking into consideration that in the majority of patients reflux of gastric juice into the laryngopharynx cannot be identified" [9].

Paroxysmal laryngospasm (LS) is defined as a suddenonset, forceful, prolonged adduction of the vocal folds resulting in glottic closure and apnea lasting at least 20 seconds. LS represents a vagally mediated reflex response of the larynx to noxious stimuli, including gastric fluid. Some reports suggest that LS may be a manifestation of GER, but only a few studies with a limited number of patients have prospectively documented GER in LS patients. Poelmans et al. [10] investigated the association of LS with GER; using endoscopy and $\mathrm{pH}$ monitoring, they demonstrated pathological GER in the vast majority of patients. Their study also suggests that LS in adult patients with unimpaired vocal fold mobility might be considered a typical supraesophageal manifestation of GER.

Severe laryngomalacia has long been associated with GER diagnosed clinically or by $\mathrm{pH}$ probe. Laryngomalacia and reflux may also be associated because both may represent effects of general low muscle tone in the upper aerodigestive tract. As reported by Manning et al. [11], 18 of 24 patients with severe laryngomalacia had a diagnosis of GER, although only 6 of the 18 had undergone a contrast study or $\mathrm{pH}$ probe.

GER is a well-known risk factor for the development of subglottic stenosis. Poetker et al. [12] reported severe reflux in 5 of 37 patients $(14 \%)$ with subglottic stenosis. This pathology may develop after thoracotomy; GER represents a risky circumstance that may worsen mucosal injuries when the patient is placed in the lateral position [13].
A causal relationship between GER and laryngeal cancer is uncertain. In a meta-analysis of the effect of GER on laryngeal cancer, Qadeer et al. [14] reported a pooled odds ratio of 2.37 for GER in patients with cancer. Vaezi et al. [15] found that the risk of laryngeal cancer is highest among patients who smoke and had GER. In this matched case-control study, the presence of GERD alone also significantly increased the risk for laryngeal cancer irrespective of smoking. A critical review of the current literature emphasized that the role of GER in laryngeal cancer remains unresolved. The high prevalence of GER could be related to the tobacco and alcohol use, but in a small proportion of cancer patients without a history of exposure to other risk factors, GER could be an independent causal factor. In laryngeal cancer, the reflux probably plays a synergistic role with other carcinogens.

That reflux is a common and important cause of chronic cough is not in dispute. GER is the second most common cause of chronic cough in immunocompetent patients who are nonsmokers, are not on angiotensin-converting enzyme inhibitors, and have normal chest radiographs [16]. The cough may be caused via vagally mediated airway hyperresponsiveness or by pulmonary microaspiration through the upper esophageal sphincter. Overnight scintigraphy performed in children with unexplained and refractory respiratory manifestations showed pulmonary aspiration, suggesting GER as a causal factor [17].

A lump in the throat (globus pharyngeus) represents a common symptom reported by patients with LPR. Nevertheless, the coexistence of globus and reflux does not mean that the reflux is the cause, and studies have reported conflicting results.

\section{Nasosinusal Manifestations}

Considering the multifactorial etiology of rhinosinusitis, GER must be considered one of the causes of chronic rhinosinusitis. A possible pathogenic mechanism is a direct action of acid reflux on respiratory nasal mucosa as on the hypopharyngolaryngeal region. So, on the nasal level, if we reject the hypothesis that reflux could directly reach paranasal sinuses through the ostia, it is possible that reflux can reach the rhinopharynx and the posterior part of the nasal cavities, where only the communicating ostium with sphenoidal sinus is present. In this way, acid reflux could lead to a mucosal nasal inflammation with subsequent edema and ostiomeatal complex obstruction. Another mechanism is the autonomic nervous system hyperreactivity induced by reflux, which then would cause nasal edema and sinusal ostium obstruction. Studies have not proven these correlations satisfactorily.

Adenoiditis is recognized as associated with upper respiratory tract infections. A relationship between adenoiditis and GER is still unknown. The question is whether the reflux promotes an inflammatory process on the adenoid tissue, or if the adenoid hypertrophy facili- 
tates the reflux, thereby modifying the inspiratory and expiratory endothoracic pressures, thus causing the retrograde movement of the gastric contents.

Few studies exist that correlate chronic sinusitis with GER. From January 1994 to December 2002, at the Pediatric Surgery Department of Siena University, we analyzed $\mathrm{pH}$-metric values of 150 subjects between 1 month and 16 years old: among them, 30 symptomatic patients younger than 1 year; 60 symptomatic patients older than 1 year (mean age: 6.7 years); 30 controls younger than 1 year; and 30 controls older than 1 year (mean age: 7 years) [2••]. Symptomatic patients presented with vomiting, regurgitation, belching, dyspepsia, and lack of appetite and were examined by an otolaryngologist and a pediatric surgeon. In our study, which was limited to patients affected with proven GER, recurrent rhinitis (not referable to allergies) and chronic rhinosinusitis were the most frequent atypical manifestations, with an incidence of $20.7 \%$ and $5.2 \%$, respectively. Thus we can state that suspicion of GER is important in pediatric subjects affected with recurrent rhinitis and chronic rhinosinusitis resistant to common treatments. Monteiro et al. [18••] support a possible correlation between chronic rhinosinusitis not associated with bronchial asthma and GER in children and adolescents, especially those presenting with typical GERD symptoms. These researchers collected clinical data suggesting that 24 -hour esophageal $\mathrm{pH}$ monitoring should be performed before indicating surgery, because $10 \%$ of chronic rhinosinusitis surgeries can be avoided.

According to Weber et al. [19], an above-average proportion of patients with chronic rhinosinusitis appear to have GERD. An international review of the literature using Medline (PubMed) was assessed; no studies showed level I evidence (randomized controlled trials). The authors believe that quantification of the association between reflux and chronic rhinosinusitis is not currently possible. In 2003, Weaver [20] showed only grade C evidence for a positive association between GER and sinusitis.

Reflux should be always regarded as a concomitant or causative factor in children with recurrent rhinitis or chronic rhinosinusitis when other etiologies have been excluded and aggressive antibiotic treatments are ineffective.

\section{Otologic Manifestations}

In animal models, eustachian tube dysfunction has been demonstrated as a result of GER, and an increasing number of studies indicate that GER may be a potential factor in the development of middle ear inflammation. The reflux can act on the middle ear both directly and indirectly. The first mechanism is the direct effect of the reflux on the mucous membrane of the ear. The indirect action consists of edema in the tube area, resulting in obstruction and impairment of the aeration of the middle ear. Nevertheless, the relationship between reflux and otitis media remains clinically controversial. In children with otitis media with effusion or recurrent otitis media, Lieu et al. [21•] investigated the putative role of GER by studying pepsin/pepsinogen in the middle ear fluid of 22 children undergoing myringotomy and tube placement for recurrent otitis media, and by querying parents about symptoms suggestive of GER. In this group of children, the incidence of reflux symptoms was similar to that in previously published studies of normal children. The investigators tested 36 samples for the presence of pepsin using the proteolytic enzyme assay and enzyme-linked immunosorbent assay (ELISA) specific for pepsinogen I. Of the 36 middle-ear samples, $67 \%$ were positive for pepsin using proteolytic assay, and $65 \%$ were positive for pepsinogen I using ELISA. Moreover, the production of pepsin and pepsinogen may be induced in the middle ear during acute infections and with chronic effusions. This endogenous production of these enzymes may reduce the pathogenetic role of GER.

To justify the hypothesis concerning the possible specific effect and significance of GER in the etiology of otitis, Velepic et al. [22] compared sequelae of chronic tubotympal disorders in children with and without GER; statistical analysis revealed that the sequelae and conductive hearing impairment were significantly higher in patients with GER. Sone et al. [23•] measured the pepsinogen levels in 60 adult patients who had otitis media with effusion. The authors concluded that the presence of pepsinogen supports the existence of GER, and that treatment for GER should be considered in patients with ear complaints, especially in those who have GER-related symptoms.

\section{Oral Cavity Lesions}

Dental and oral mucosa erosions have been described in bulimic patients, in patients with hiatus hernia, and in children with GER. Teeth erosion is considered the main GER manifestation in the oral cavity [24]. Acid exposure causes demineralization of the enamel, which can progress to the subsurface layers and result in complete loss of the dental tissue. Involuntary and unexpected reflux occurring during sleep allows the posterior teeth to be in contact with the least diluted acid refluxate compared with the anterior teeth, which are exposed to acid fluid buffered with saliva; in this instance, the pattern of erosion shows an involvement mainly of the posterior teeth. However, the published studies are uncontrolled and data sometimes show the opposite. The role of GER as a causative factor in dental erosion remains speculative. Mamede et al. [25] found a few correlations between the hypertrophy of the tongue base and GER.

\section{Conclusions}

Among ENT patients, $4 \%$ to $10 \%$ have "hidden" GERD, but a true association between LPR and ENT manifestations is difficult to prove. Rhinosinusitis, otitis media, 
and laryngeal and oral pathologies all have multiple risk factors, making it difficult to isolate the effect of a single factor. Identification of GER as an independent causal factor in ENT manifestations can sometimes be difficult. GER has no standard definition or method of diagnosis. Therefore, all types of reflux (acid, nonacid, liquid, mixed, and air) detected by impedance changes should probably be considered. In 24-hour $\mathrm{pH}$ monitoring with dual or triple electrodes, the $\mathrm{AUCH}^{+}$is the best parameter to evaluate GER in adults and children, but is not always considered. Similarly, the definition and diagnostic accuracy of ENT pathologies vary with the examiner, exam accuracy, and the other tests used (eg, CT or tympanogram). Independent associations of GER with awake apnea, reactive airway disease, and recurrent pneumonia have been demonstrated. The overall summary grade of evidence for a negative association between GER and otitis media is grade $\mathrm{C}$. There is also grade $\mathrm{C}$ evidence for a positive association between GER and sinusitis. Any causal relationship between ENT manifestations and GER is therefore controversial. Further well-designed, prospective, large-scale trials are warranted.

\section{Disclosures}

No potential conflicts of interest relevant to this article were reported.

\section{References and Recommended Reading}

Papers of particular interest, published recently, have been highlighted as:

- Of importance

$\bullet \quad$ Of major importance

1.• Friedman M, Friedman M, Schalch P, et al.: Wireless upper esophageal monitoring for laryngopharyngeal reflux (LPR). Otolaryngol Head Neck Surg 2007, 137:471-476.

At present, a firm connection between GER and ENT pathologies remains controversial due to the lack of standardized diagnostic methods for GER. The value of this study and other studies

$[2 \bullet \bullet, 6 \bullet \bullet, 18 \bullet \bullet]$ is the attempt to standardize GER diagnosis by introducing new methods or parameters.

2.• Caruso G, Passàli FM: ENT manifestations of gastrooesophageal reflux in children. Acta Otorhinolaryng Ital 2006, 26:252-255.

A firm connection between GER and ENT pathologies remains controversial because standardized methods for GER diagnosis are needed.

This study is valuable for attempting to standardize GER diagnosis via new methods or parameters. (Also see $[1 \bullet \bullet, 6 \bullet \bullet, 18 \bullet \bullet]$.)

3. Morice AH: Is reflux cough due to gastroesophageal reflux disease or laryngopharyngeal reflux? Lung 2007, Epub ahead of print.

4. $\quad$ Groome M, Cotton JP, Borland M, et al.: Prevalence of laryngopharyngeal reflux in a population with gastroesophageal reflux. Laryngoscope 2007, 117:1424-1428. By examining a large number of patients, study investigators clearly demonstrated the correlation between the severity of GERD and the prevalence of LPR symptoms. LPR and GERD can thus be considered common and interlinked conditions.

5. Mesallam TA, Stemple JC, Sobeih TM, et al.: Reflux symptom index versus reflux finding score. Ann Otol Rhinol Laryngol 2007, 116:436-440.
6.• Vázquez de la Iglesia F, González SF, de la Cámara Gómez $\mathrm{M}$ : Reflujo faringolaríngeo: correlación entre los síntomas y los signos mediante cuestionarios de valoración clínica y fibroendoscópica. Es suficiente para realizar el diagnóstico? Acta Otorrinolaringol Esp 2007, 58:421-425.

A firm connection between GER and ENT pathologies remains controversial because GER diagnostic methods are not standardized. The value of this study and others $[1 \bullet \bullet, 2 \bullet \bullet, 18 \bullet \bullet]$ is the attempt to standardize GER diagnosis by using new methods or parameters.

7. Hickson C, Simpson CB, Falcon R: Laryngeal pseudosulcus as a predictor of laryngopharyngeal reflux. Laryngoscope 2001, 111:1742-1745.

8. Belafsky PC, Postma GN, Koufman JA: The association between laryngeal peudosulcus and laryngopharyngeal reflux. Otolaryngol Head Neck Surg 2002, 126:649-652.

9. Joniau S, Bradshaw A, Esterman A, et al.: Reflux and laryngitis: a systematic review. Otolaryngol Head Neck Surg 2007, 136:686-692.

10. Poelmans J, Tack J, Feenstra L: Paroxysmal laryngospasm: a typical but underrecognized supraesophageal manifestation of gastroesophageal reflux? Dig Dis Sci 2004, 49:1868-1874.

11. Manning SC, Inglis AF, Mouzakes J, et al.: Laryngeal anatomic differences in pediatric patients with severe laryngomalacia. Arch Otolaryngol Head Neck Surg 2005, 131:340-343.

12. Poetker DM, Ettema SL, Blumin JH: Association of airway abnormalities and risk factors in 37 subglottic stenosis patients. Otolaryngol Head Neck Surg 2006, 135:434-437.

13. Lin BF, Lee SC, Kao CH, et al.: Subglottic stenosis after thoracotomy: a case report. Acta Anaesthesiol Taiwan 2007, 45:59-62.

14. Qadeer MA, Colabianchi N, Strome M: Gastroesophageal reflux and laryngeal cancer: causation or association? A critical review. Am J Otolaryngol 2006, 27:119-128.

15. Vaezi MF, Qadeer MA, Lopez R: Laryngeal cancer and gastroesophageal reflux disease: a case-control study. Am J Med 2006, 119:768-776.

16. Chandra KM, Harding SM: Therapy insight: treatment of gastroesophageal reflux in adults with chronic cough. Nat Clin Pract Gastroenterol Hepatol 2007, 4:604-613.

17. Ravelli AM, Panarotto MB, Verdoni L, et al.: Pulmonary aspiration shown by scintigraphy in gastroesophageal refluxrelated respiratory disease. Chest 2006, 130:1520-1526.

18.• Monteiro VR, Sdepanian VL, Weckx L, et al.: Twenty-fourhour esophageal $\mathrm{pH}$ monitoring in children and adolescents with chronic and/or recurrent rhinosinusitis. Braz J Med Biol Res 2005, 38:215-220.

A firm connection between GER and ENT pathologies remains controversial because GER diagnostic methods are not standardized. The value of this study and others $[1 \bullet \bullet, 2 \bullet \bullet, 6 \bullet \bullet$ is the attempt to standardize GER diagnosis by introducing new methods or parameters.

19. Weber RK, Jaspersen D, Keerl R, et al.: Gastroesophageal reflux disease and chronic sinusitis. Laryngorbinootologie 2004, 83:189-195.

20. Weaver EM: Association between gastroesophageal reflux and sinusitis, otitis media and laryngeal malignancy: a systematic review of evidence. Am J Med 2003, 115(Suppl 3A):815-895.

21. Lieu JE, Muthappan PG, Uppaluri R: Association of reflux with otitis media in children. Otolaryngol Head Neck Surg 2005, 133:357-361.

This study investigates the role of GER in otitis media in children. It reports possible pathogenetic mechanisms, and a direct relation is supposed, although not confirmed, by pepsin and pepsinogen levels in middle ear effusion. A study was also conducted in adults $[23 \bullet]$.

22. Velepic MM, Velepic MS, Starcevic R, et al.: Gastroesophageal reflux and sequelae of chronic tubotympanal disorders in children. Acta Otolaryngol 2004, 124:914-917. 
23.• Sone M, Yamamuro Y, Hayashi H, et al.: Otitis media in adults as a symptom of gastroesophageal reflux. Otolaryngol Head Neck Surg 2007, 136:19-22.

This study investigates the role of GER in otitis media in adults. The possible pathogenetic mechanisms are reported and a direct relation is supposed, but not confirmed, by pepsin and pepsinogen levels in middle ear effusion. A study was also conducted in children [21•].
24. Cebrián-Carretero JL, López-Arcas-Calleja JM: Gastroesophageal reflux diagnosed by occlusal splint tintion. Med Oral Patol Oral Cir Bucal 2006, 11:E26-E28.

25. Mamede RC, De Mello-Filho FV, Dantas RO: Severe hypertrophy of the base of the tongue in adults. Otolaryngol Head Neck Surg 2004, 131:378-382. 
Copyright of Current Allergy \& Asthma Reports is the property of Springer Science \& Business Media B.V. and its content may not be copied or emailed to multiple sites or posted to a listserv without the copyright holder's express written permission. However, users may print, download, or email articles for individual use. 